time interval was too short for them to be screened for TB. Abstract S41 table 1 illustrates the differences between NICE and BHIVA strategies over the 10-year period. Neither screening method had optimal sensitivity and specificity. Both guidelines' eligible groups were only twice as likely to develop active TB compared to those ineligible for screening. NICE guidelines were poorly specific while the BHIVA strategy missed $42 \%$ of cases (largely as they were either UK born or on ART). Using data from our UK HIV population, we find that new TB screening guidance does not appear to discriminate those at risk of active $\mathrm{TB}$.

\section{S42 EARLY IDENTIFICATION OF MYCOBACTERIUM TUBERCULOSIS (M.tb) AND MYCOBACTERIUM OTHER THAN TUBERCULOSIS (MOTT) BY "CORD FORMATION" EVALUATION IN LIQUID CULTURE}

doi:10.1136/thoraxjnl-2011-201054b.42

A Elsaghir, S Abdullah, F Sundram, D D Creer. Barnet and Chase Farm Hospitals NHS Trust, London, UK

The identification of M.tb in smear negative samples has the inherent TB culture delays. These delays potentially affect treatment choices and contact tracing decisions particularly where TB prevalence is low and isolation of MOTT common. Faster molecular techniques are expensive and not universally available. The identification of cord formation ${ }^{1}$ in positive cultures gives an early indication of the likely $\mathrm{TB}$ culture result before the final reference laboratory identification (local audit data: M.tb 12.3 \pm 5.9 d, MOTT $13.7 \pm 11.6 \mathrm{~d}$ ). We evaluated the presence of cord formation in AFB smears prepared from MGIT tubes and stained by auramine-phenol staining from positive liquid culture (BACTEC MGIT growth supplement medium) for the presumptive identification of $M . t b$. We prospectively evaluated 612 positive mycobacterial culture specimens from 316 patients over 3 years. All specimens were sent to our local reference laboratory for species identification. The identification of $M . t b$ by the presence of cord formation was compared with the subsequent reference laboratory report. 420/426 samples showing cording by smear microscopy were confirmed by the reference laboratory as M.tb (PPV: 98.6\%). 182/186 non-cording culture positive specimens were confirmed as MOTT (NPV: 97.9\%). The $6 / 612(0.9 \%)$ MOTT misidentified as $M . t b$ by cording were $M$ kansasii as previously described. ${ }^{1}$ The $4 / 612(0.7 \%)$ M.tb misidentified as MOTT by the absence of cording were all $3+$ sputum smear positive samples as previously described. ${ }^{2}$ The presumptive identification of $M . t b$ and MOTT by the presence or absence of cording formation in liquid culture is both sensitive and specific. This technique has a potentially significant impact on treatment timing and contact tracing decisions.

\section{REFERENCES}

1. McCarter YS, et al. Cord formation in BACTEC medium is a reliable, rapid method for presumptive identification of Mycobacterium tuberculosis complex. J Clin Microbiol 1998;36:2769-71.

2. Koksalan $\mathbf{O K}$, et al. Reliability of cord formation in BACTEC 12B/13A media for presumptive identification of Mycobacterium tuberculosis complex in laboratories with a high prevalence of Mycobacterium tuberculosis. Eur J Clin Microbiol Infect Dis 2002;21:314-17.

\section{S43 POLYFUNCTIONAL T CELLS REVEAL THE SPECTRUM OF TUBERCULOSIS IN HIV CO-INFECTION THROUGH THE IDENTIFICATION OF IMMUNOLOGICAL CORRELATES OF LATENT AND ACTIVE DISEASE}

doi:10.1136/thoraxjnl-2011-201054b.43

${ }^{1} \mathrm{~K}$ M Pollock, ${ }^{1} \mathrm{D}$ Montamat-Sicotte, ${ }^{2} \mathrm{G}$ Cooke, ${ }^{1} \mathrm{M}$ Kapembwa, ${ }^{3} \mathrm{O} \mathrm{M}$ Kon, ${ }^{2} \mathrm{G}$ P Taylor, ${ }^{1} \mathrm{~A}$ Lalvani. ${ }^{1}$ Department of Respiratory Medicine, Tuberculosis Research Unit, National
Heart and Lung Institute, Imperial College London, London, UK; ${ }^{2}$ Department of Medicine, Division of Infectious Diseases, Imperial College London, London, UK ${ }^{3}$ Tuberculosis Service, St Mary's Hospital, Imperial College Healthcare Trust, London, UK

Background Approximately one-third of the world's population is infected with Mycobacterium tuberculosis (MTB), mostly as latent tuberculosis infection (LTBI). HIV co-infection confers the single greatest risk of progression to active tuberculosis (TB) and thus provides an opportunity to study the host-pathogen interaction in LTBI. The stage of TB disease and effect of advancing HIV infection should be reflected in the phenotype and function of MTB-specific T cells.

Aims To compare MTB-specific CD4+ and CD8+ Tcell responses in donors from four clinical phenotypes (TB/HIV, TB, LTBI/HIV and LTBI) and test whether TB in the context of HIV is a spectrum of disease dependent on mycobacterial load consequent upon advancing immunosuppression and viraemia.

Methods Donors $(n=34)$ with or without HIV co-infection were carefully selected for active TB or LTBI. Peripheral blood mononuclear cells (PBMCs) were stimulated overnight with purified protein derivative (PPD) or a combination of MTB-specific antigens. An 11-colour intracellular cytokine secretion assay was used to assess CD3, CD4, CD8, IFN- $\gamma$, IL-2 and TNF- $\alpha$ expression.

Results The proportion of participants with a CD8+ PPD-specific IFN- $\gamma(p=0.001)$ or TNF- $\alpha(p=0.006)$ response closely mirrored stage of TB disease, whereas the equivalent CD4 response was unaffected. The proportion of $\mathrm{CD} 4+$ and $\mathrm{CD} 8+\mathrm{PPD}$-specific cells increased with TB stage for IFN- $\gamma$ and TNF- $\alpha$, but did not increase in HIV/TB co-infection compared with TB (Abstract S43 figure 1). When individual cytokine subsets were examined, the percentage of
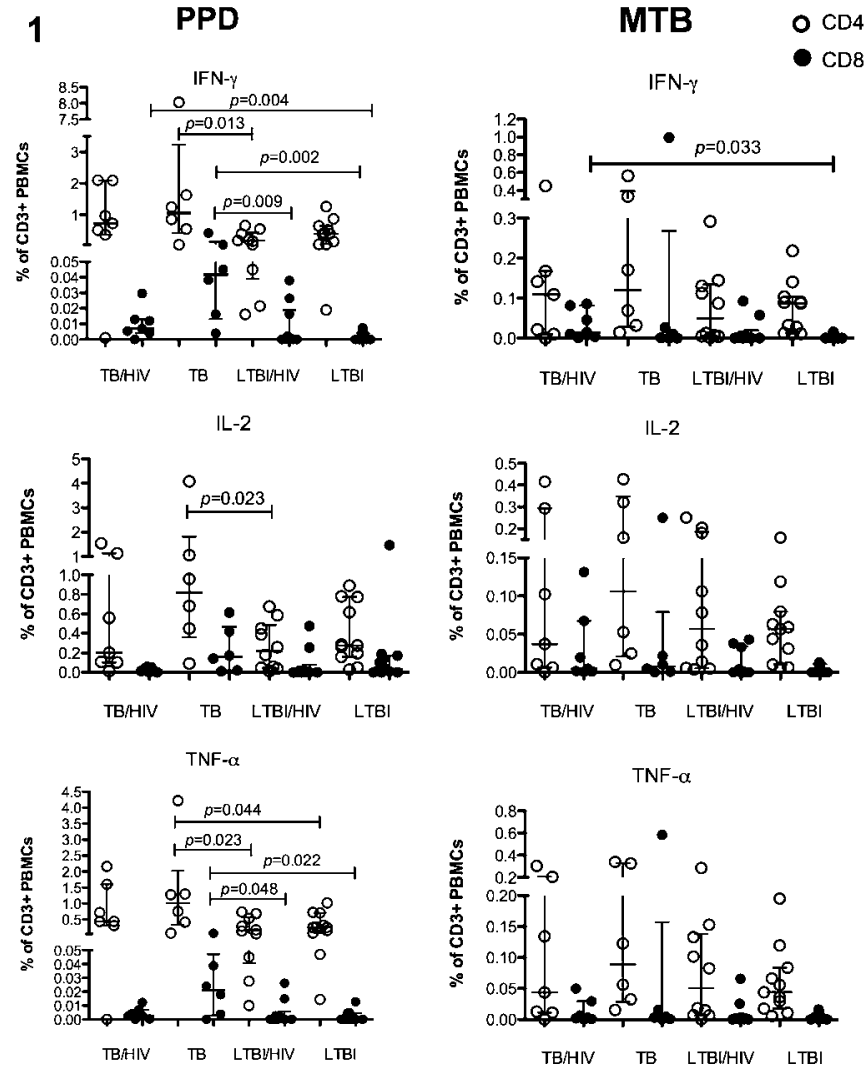

Abstract S43 Figure 1 The proportion of CD3 + CD4+ and CD8+ cells with a total cytokine response to PPD or MTB antigens following $16 \mathrm{~h}$ of stimulation. PBMCs were stained with an 11-colour panel including a dead cell marker and fluorochrome-conjugated antibodies for CD3, CD4, CD8, IFN- $\gamma$, IL-2 and TNF- $\alpha$ and acquired on an LSR-II flow cytometer. 